\title{
X-ray histotomography
}

Characterising every cell of a whole organism

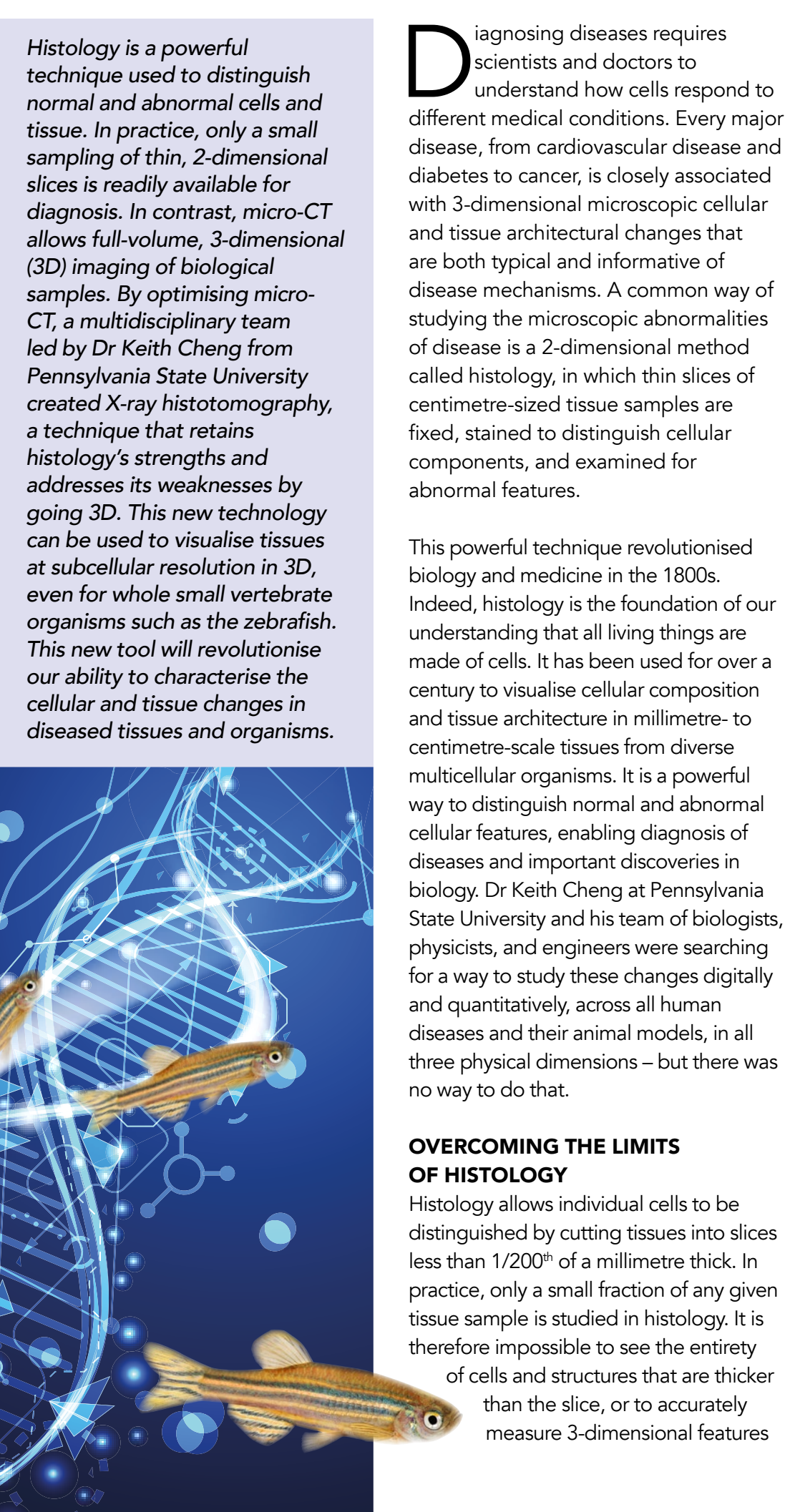

such as shape or volume. An additional mitation is that physical sectioning is irreversible, making it impossible to Physical cunting introduces tissue loss and distorions that compromise our ability to distortions that compromise our ability to
digitally define the $3 \mathrm{D}$ structure of tissue digitally define the 3D structure of tissue and organisms at the cellular level.

To circumvent histology's limitations, whole-sample, 3D version of with comprehensive quantitative and volumetric measurements of scientifically and clinically meaningful features, A 3D histology would enable safer drugs, more precise diagnoses, and more in-depth knowledge of the interactive roles of genes, chemicals, and disease in biology.

MICRO-COMPUTED TOMOGRAPHY Larger internal structures within the

human body are routinely visualised using a technique known as computed tomography, CT for short. CT scan imaging is widely used in hospitals. This technique uses X-rays and a computer to create detailed pictures of the inside of the body. Ilt takes $X$-ray pictures from thedres to thousands of different angles "Micro-CT" works on the same pringiples as CT scans. While CT scans allow us to see things as small as a millimetre, microCT allows us to see things 1000-times smaller - even parts of cells. To achieve 3D histology, both high tissue contrast and high resolution are needed to distinguish and characterise cells and tissues. Since contrast is insufficient in unstained samples, specific metal-based stains are needed for histology-level contrast. The samples for micro-CT can be insects, tissues, vertebrate embryos and zebrafish at any life stage.

\section{MICRO-CT OPTIMISATION}

deally, 3D histology would allow one to rapidly scan for abnormalities in entire

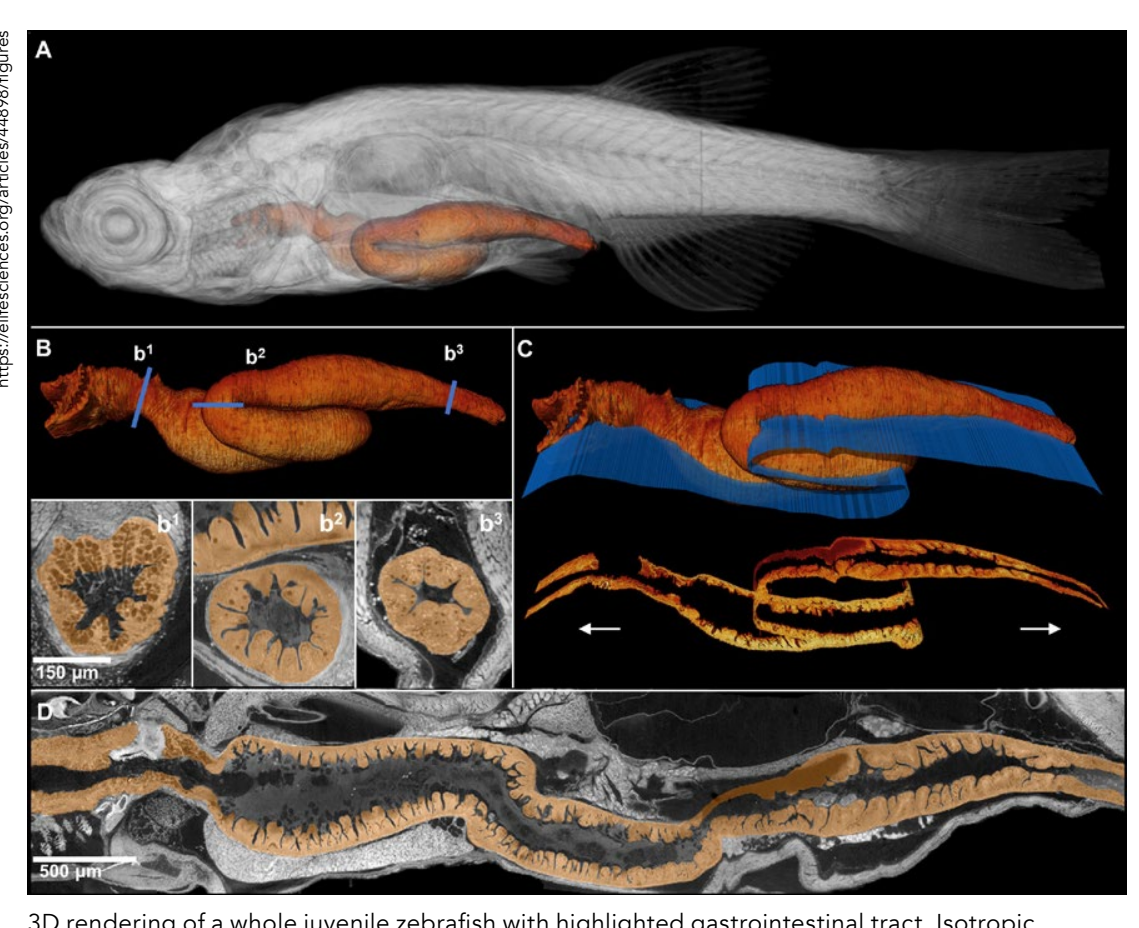

3 D rendering of a whole juvenile zebrafish with highlighted gastrointestinal tract. Isotropic
resolution of data permits virtual slicing at any angle without distortion or a decrease in resolution.

the same time, enable the histological examination of any portion of the sample micro-CT by optimising X-ray op
from any angle. No existing method has sample preparation, and digital

had the necessary combination of field of

view, resolution and contrast. That was,

until Dr Cheng and his team adapted

lens and camera technologies from the

semiconductor and imaging industries.

Implementing these improvements would

allow scientists and doctors to image

even whole small organisms at subcellular

resolution.

X-RAY HISTOTOMOGRAPHY

By optimising micro-CT, Dr Cheng and his workflow. The resulting adjustments for high throughput (the speed of data generation and collection). Samples are rigidly embedded in plastic so that they can be preserved for years without deterioration and re-imaged for discovery, validation, and reproducibilly. Their system also reduces unwanted sample movement during scanning resulting in improved inage quality team created a 3D histology they called micro-CT system, the Penn State team vertebrate model with diverse tissues, and about $70 \%$ of human genes have at 政 as a vertebrate model of human biology and disease.

With X-ray histotomography, Dr Cheng and his team were able to obtain 3D digital reconstructions of whole zebrafish These digital zebrafish allow tissues and cells to be visualised across organ systems histology and histotomography have the resolution needed to distinguish cellular features in $2 \mathrm{D}$ slices, only the latter reveads elongated, complex 3D tissue structures such as vessels, heve tracts, and bones. The digital representation of full volumes " tissue has created a opportunity for " which we can digitaly "cut" and " in the tissue at any angle or slice thickness. Parameters can be customised to easily visualise various tissue structures. Sructures that are normally inaccessible X-ray histotomography. For example, the team was able to study features of complex structures such as the gills and the gut.

\section{COMPUTATIONAL PHENOTYPING} FOR PHENOMICS

The intention of Dr Cheng's work was to fill a critical gap in developing the transdisciplinary field called phenomics. Phenomics is the study of complete sets of phenotypes across all organism organisms the resservable traits of

result from interaction tween genes,

as part of a vision

Dr Cheng and his team were searching environment, disease, quantitatively compare for a way to study cellular changes physical feature is digitally and quantitatively, across all insenotype; for colour is a phenotype $\begin{array}{lll}X \text {-ray histotomography cell types in a whole animal model. } & \begin{array}{l}\text { colour is a phenotype } \\ \text { that depends on our }\end{array}\end{array}$ can achieve the

theeded a model that had the full range required for histological evaluations and of tissue complexity and sample sizes the ability to phenotype all cell types and commonly encountered in experimenta tissues in whole organisms. This improved form of micro-CT retains the strengths of histology and creates a path to digita histology for full sample volumes.

A primary goal was to implement a technology that can enable large-scale 3D phenotyping. X-ray

\section{and clinical histology.}

Multiple factors make the zebrafis a useful model for developing whole-organism 3D tissue imaging. Zebrafish measure one millimetre to the zebrafish is a in with. Indeed stress, or temperature and humidity are examples of environmental factors that can impact phenotypes. Every symptom caused by disease is also a phenotype. The purpose of the emerging field of phenomics is to systematically determine across biology to understand the links disease factors. measure, and compare phenotypes between specific phenotypes and the 
Phenotyping consists of recording the dbserved features of an organism. It is medrine since phe in biology and dentifying symptoms to make a diagnosis, and to understand sets mutant phenotypes that illuminate the functions of a gene. Because of the mportance of histology. Dr Cheng and his team sought to expand its power in phenotyping in the third dimension. In this way, 3D data of cell and tissue architecture would be used to systematically study how disease, genetics and environmental factors mpact organismal phenotypes. 3D microanatomical phenotyping would also improve the accuracy and prognostic implications of diagnoses that can benefit from quantification of histological details. Because phenotypes often ffect more than one organ system, the phenotypes across all cell types makes ideal for determining phenotypes more completely. Examining every cell type in a whole organism is an ideal way to study the phenotype at both organismal (organ sizes and shapes) and cellular scales. Justifiably, Dr Cheng and a growing team of collaborators are excited about working together towards a whole-organism 3D imaging technique at subcellular resolution and to create scientifically and medically useful computational tools to detect and interpret those changes.

MULTIPLE POSSIBILITIES

AND FUTURE DIRECTIONS

The combing

histotomography to en able visualisation of

convoluted, millimetre- to centimetre caf

structures such as intestines, bones, blood

vessels, and nenve tracts, while retaining

the ability to examine cellular change at

subcellular resolution. Histological features are usually described qualitatively, but the most useful measures in science are quantitative. X-ray histotomography solves this problem by making it possible to characterise tissue change quantitatively. Histotomography's unprecedented combination of visualisation of all cell types, high resolution and scale, allow users to analyse cellular features such as sze and shape in the context of the whole sample. For example, densities of brain in colour maps that revealed striking
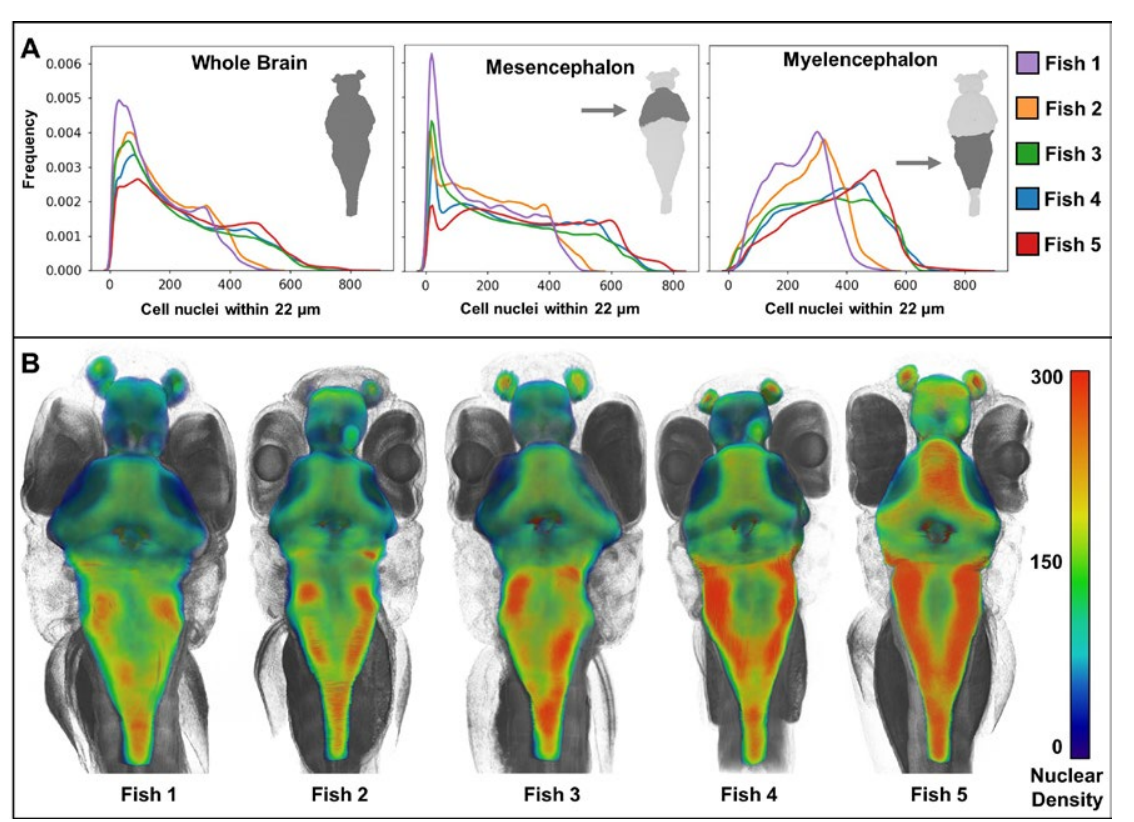

Heat maps of regional density of brain cell nuclei reveal striking phenotypic variation between

X-ray histotomography may soon be used to study changes across hundreds of cell types in centimetre-sized organisms or tissue samples.

individual phenotypic variation between
zebrafish siblings born on the same day.

Dr Cheng and his team have also

confirmed that histotomography can be used to identify pathological phenotyp and make diagnoses. Indeed, minute histopathological features such as nuclear fragmentation have been detected in DNA synthesis The a structures such as the ai bledder and its tiny preumatic duct cannot be confirmed using histology due to their small size and tortuous shape. Because histotomograp hy

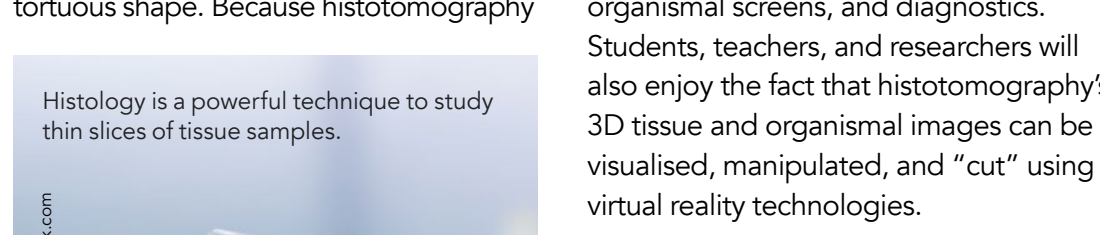

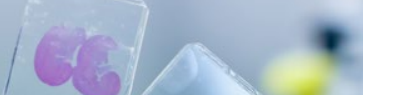

lows visualisation of the entire organism cxquiste detail, they were able to forms those structures, but also that their cells share suctures, but also that their such as nuclear atypia.

X-ray histotomography could be used to study changes across hundreds of cell sized organism or tisue centimetre-

computational and visual insights into 3D

cell and tissue architecture will be useful

for reference atlases, comprehensive

\section{Current efforts aim at increasing}

resolution, field of view, and imaging

speed, and automating phenotyping

and tissue diagnosis. The enabled

computational tissue analysis will lead

to a better understanding of how genes
and the environment contribute to

and the environment contribute to

environment and safer drugs.

\section{(3) Behind the Research}

3) .4 Dr Keith C. Cheng

IEVA: kcheng76@gmail.com W: chenglab.io

\section{Research Objectives}

Dr Cheng's team has created X-ray histotomography, the first 3D imaging method that allows digital cell and tissue diagnostics fom entire tissue samples and small organisms.

\section{Detail}

Address

500 University Drive, Rm 7866

Hershey, PA 17033

USA

Bio

Keith Cheng earned a BA in Biochemical Sciences from Harvard, MD at NYU, PhD in Molecular Genetics at the University of Washington, and tranedin Anatomic Pathology

Washington. He is Director of the Computational Phenomics Initiative at Penn State, and his research encompasses cancer, genetics and

\section{Funding}

Ofice of Research Infrastructure and Programs, $\mathrm{NIH}$ Research

- Pennsylvania State Tobacco
Funds - PSU/Penn State College of Medicine te for Computationa and Data Science (ICDS) Department of Pathology, Penn State

Collaborators - Patrick La Riviere - Steven Yuxing Wan - Xlanghuixiao

\section{References}

Ding, Y., Vanselow, D., Yakovlev, M., Katz, S., Lin, A., Clark, D., Vargas, P., Xin, X., Copper, J., Canfield, V., Ang, K., Wang, Y., Xiao, X., Carlo, F.D., van Rossum, D.B., Riviere, P.L., and Cheng, K.C. (2019). Computational $3 \mathrm{D}$ histological phenotyping of

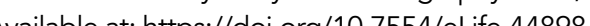

Lin, A.Y., Ding, Y., Vanselow, D.J., Katz, S.R., Yakovlev, M.A., Clark, D.P., Mandrell, D., Copper, J.E., van Rossum, D.B., and Cheng, K.C. (2018). Rigid Embedding of Fixed and Stained, Whole, Millimeter-Scale Specimen for Section-free 3D Histology by Micro-Computed (140), 58293. Available at: https://doi.org/10.3791/58293

Xin, X., Clark, D., Ang, K.C., van Rossum, D.B. Copper, J., Xlao, X., La Riviere, P.J., and Cheng, K.C. (2017). Synchrotron microCT imaging of soft tissue in juvenile zebrafish reveals retinotectal projections. Proceedings of SPIE--the International Society for Optical Engineening, 10060, 100601. Available at: https://doi, org/10.1117/12.2267477

Cheng, K.C., Katz, S.R., Lin, A.Y., Xin, X., and Ding, Y. (2016) Whole-Organism Cellular Pathology: A Systems Approach at: https://doi.org/10.1016/bs.adgen.2016.05.003

\section{Personal Response}

What inspired you to believe that optimising micro-CT

II We reasoned that tissue staining that has allowed scientists and physicians to recognise all cell types could
be achieved in $3 \mathrm{D}$, and that advances in computer and digital imaging technologies and existing technor in chip manufacturing could be combined with $3 \mathrm{D}$ tissue staining to enable a $3 \mathrm{D}$ histology for pencil-wide
organisms and tissue samples.

How will you apply X-ray histotomography

II We are expanding the use of $X$-ray histotomography and to compare those structures and changes visually and quantitatively. We are, for example, testing applications of histotomography to toxicology, and towards a better understanding of the effect of COVID-19 in all organ systems. By automating segmentation and analysis, the phenotypes will be a part of a 'computational phenomics'

PennState

College of Medicine 